\title{
The Rolling of a Homogeneous Ball with Slipping on a Horizontal Rotating Plane
}

\section{T. B. Ivanova}

This paper is concerned with the rolling of a homogeneous ball with slipping on a uniformly rotating horizontal plane. We take into account viscous friction forces arising when there is slipping at the contact point. It is shown that, as the coefficient of viscosity tends to infinity, the solution of the generalized problem on each fixed time interval tends to a solution of the corresponding nonholonomic problem.

Keywords: rotating surface, turntable, nonholonomic constraint, rolling ball, sliding, viscous friction

\section{Introduction}

This paper investigates the motion of a dynamically symmetric (in particular, homogeneous) ball rolling with slipping on a uniformly rotating horizontal plane.

In the case where there is no slipping, the motion of the ball is subject to an inhomogeneous nonholonomic constraint, which corresponds to the condition that the velocities of the contacting points on the surface of the ball and the rotating plane be the same. In this case, the system is linear and admits explicit integration (see, e.g., [1-3] and references therein). The trajectories of the center of mass of the ball relative to a fixed reference system are circles, and the ball moves with constant angular velocity, which depends only on the angular velocity of the surface on which the ball rolls, and on the radius of inertia of the ball.

Received April 12, 2019

Accepted May 28, 2019

This work is supported by the Russian Science Foundation under grant 19-71-30012 and performed in Steklov Mathematical Institute of Russian Academy of Sciences.

Tatiana B. Ivanova

tbesp@rcd.ru

Steklov Mathematical Institute, Russian Academy of Sciences,

ul. Gubkina 8, Moscow, 119991 Russia

RUSSIAN JOURNAL OF NONLINEAR DYNAMICS, 2019, 15(2), 171-178 
On the other hand, when the ball moves, for example, on a fast rotating plane, slipping may be observed at the contact point. In this case, at the point of contact with the plane, from the plane the ball is acted upon not only by the normal reaction, but also by the friction force.

In this paper, we assume that the friction force is viscous, that is, it depends linearly on the velocity of the contact point relative to the rotating plane and has an opposite direction.

Taking into account viscous friction forces leaves the problem linear and, as in the nonholonomic case, the system can be explicitly integrated. In addition, as the coefficient of viscosity tends to infinity, the solution on each fixed time interval tends to a solution of the corresponding nonholonomic problem.

We note that a similar result was proved in [4] for a Chaplygin sleigh executing inertial motion on a horizontal plane, and in [5] for a ball moving on the surface of a rotating cylinder. The correctness of such a passage to the limit from dissipative holonomic systems to conservative nonholonomic systems in general form is discussed in $[6,7]$.

The theoretical results obtained supplement the models of motion of a ball without slipping on a rotating plane [2]. The problem under consideration is of great importance for applications. On the one hand, the dynamics of the ball with slipping and viscous friction is described by a linear system of differential equations which is investigated by standard methods of linear algebra and of the theory of ordinary differential equations. On the other hand, the motion of the ball with slipping on a plane can be observed in an experiment. Using high-speed cameras and modern methods of processing experimental results, one can verify the adequacy of the friction models used.

\section{Equations of motion of a ball moving with slipping on a rotating plane}

Consider the motion of a homogeneous ball of mass $m$ and radius $R$ with slipping on a horizontal rough plane. The plane rotates with constant angular velocity $\Omega$ about the axis coinciding with the external normal to the plane (Fig. 1).
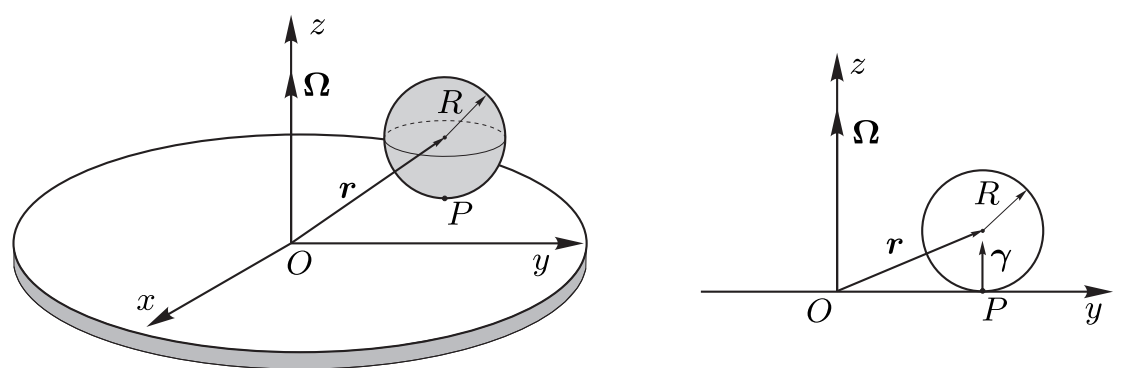

Fig. 1. A schematic model of a ball on a rotating plane. In the left figure, the axis $O x$ is perpendicular to the figure plane and is directed toward the reader.

To describe the dynamics of the ball, we define a fixed (inertial) coordinate system $O x y z$ in such a way that the plane $O x y$ is parallel to the plane of motion of the center of mass of the ball and the axis $O z$ is directed along the external normal $\boldsymbol{\gamma}$ to the plane of motion, $\boldsymbol{g}=(0,0,1)$.

In the chosen coordinate system, the position of the center of mass of the ball is defined by the radius vector $\boldsymbol{r}=(x, y, z)$, the free-fall acceleration vector has the form $\boldsymbol{g}=(0,0,-g)$, and the angular velocity of the rotating plane on which the ball rolls is $\boldsymbol{\Omega}=(0,0, \Omega)$ (Fig. 1). 
In this paper, we assume that the ball moves without loss of contact with the plane $(z \equiv R)$, with slipping at the point of contact. At the contact point, the ball is acted upon by the viscous friction force:

$$
\boldsymbol{F}_{f}=-\mu_{0} \boldsymbol{u},
$$

where $\mu_{0}$ is the coefficient of viscous friction, $\boldsymbol{u}$ is the velocity of the contact point on the ball relative to the rotating plane.

Let $\boldsymbol{v}$ denote the velocity of the center of mass of the ball relative to the fixed coordinate system:

$$
v=\dot{r}
$$

Changes in the linear and angular momenta of the ball relative to its center are described by the Newton - Euler equations:

$$
m \dot{\boldsymbol{v}}=-\mu_{0} \boldsymbol{u}+m \boldsymbol{g}+\boldsymbol{N}, \quad I \dot{\boldsymbol{\omega}}=\mu_{0} R \boldsymbol{\gamma} \times \boldsymbol{u},
$$

where $I$ is the central tensor of inertia of the ball, $\boldsymbol{\omega}$ is the angular velocity of the ball, and $\boldsymbol{N}=(0,0, N)$ is the reaction force acting from the plane and directed along the normal to it.

The velocity $\boldsymbol{u}$ of the contact point located on the ball is given by

$$
\boldsymbol{u}=\boldsymbol{v}+R \boldsymbol{g} \times \boldsymbol{\omega}-\boldsymbol{\Omega} \times \boldsymbol{r} .
$$

In what follows, we will write all variables and equations in dimensionless form. To this end, we take the radius of the ball $R$ as the unit of distance, the quantity $1 / \Omega$ as the unit of time, and the mass of the ball $m$ as the unit of mass, that is, in Eqs. (1.1)-(1.4) we make the following change of variables:

$$
\begin{gathered}
\frac{\boldsymbol{r}}{R} \rightarrow \boldsymbol{r}, \quad t \Omega \rightarrow t, \quad \frac{\boldsymbol{v}}{R \Omega} \rightarrow \boldsymbol{v}, \quad \frac{\boldsymbol{u}}{R \Omega} \rightarrow \boldsymbol{u}, \quad \frac{\omega}{\Omega} \rightarrow \omega, \\
\frac{g}{R \Omega^{2}} \rightarrow g, \quad \frac{N}{m R \Omega^{2}} \rightarrow N, \quad \frac{\mu_{0}}{m \Omega} \rightarrow \mu_{0} .
\end{gathered}
$$

Substituting the expression for $\boldsymbol{u}$ (1.4) into the system (1.2)-(1.3), we obtain a closed system of nine differential first-order equations (in dimensionless variables):

$$
\begin{aligned}
\dot{\boldsymbol{r}} & =\boldsymbol{v}, \\
\dot{\boldsymbol{v}} & =-\mu_{0}(\boldsymbol{v}+\boldsymbol{\gamma} \times \boldsymbol{\omega}-\boldsymbol{\gamma} \times \boldsymbol{r})+\boldsymbol{g}+\boldsymbol{N}, \\
\dot{\boldsymbol{\omega}} & =\frac{\mu_{0}}{k}(\boldsymbol{\gamma} \times \boldsymbol{v}-\boldsymbol{\omega}+\gamma(\boldsymbol{\gamma} \cdot \boldsymbol{\omega})+\boldsymbol{r}-\gamma(\boldsymbol{\gamma} \cdot \boldsymbol{r})),
\end{aligned}
$$

where $k=I /\left(m R^{2}\right)$ and $k=2 / 5$ for a homogeneous ball.

According to the condition of motion without loss of contact, $\dot{z} \equiv 0$. Then, projecting the first equation of (1.6) onto the axis $O z$, we find $v_{z}=0, \dot{v}_{z}=0$. Thus, from the projection of the second equation of (1.6) onto the axis $O z$ we find the reaction force

$$
N=g \text {. }
$$

The projection of the third equation of (1.6) onto the axis $O z$ has the form

$$
\dot{\omega}_{z}=0,
$$

that is, the vertical projection of the angular velocity of the ball remains unchanged. 
We finally find that the system of equations (1.6) reduces to a linear system of six differential first-order equations relative to the vector of the variables

$$
\boldsymbol{z}=\left(x, y, v_{x}, v_{y}, \omega_{x}, \omega_{y}\right),
$$

which can be represented in matrix form

$$
\dot{z}=\mathbf{L} z, \quad \mathbf{L}=\left(\begin{array}{ccc}
\mathbf{0} & \mathbf{I} & \mathbf{0} \\
\mu \mathbf{J} & -\mu \mathbf{I} & -\mu \mathbf{J} \\
\frac{\mu}{k} \mathbf{I} & \frac{\mu}{k} \mathbf{J} & -\frac{\mu}{k} \mathbf{I}
\end{array}\right),
$$

where $\mathbf{L}$ is a $6 \times 6$ block matrix, $\mathbf{I}$ is a $2 \times 2$ identity matrix, and $\mathbf{J}_{i j}=-\varepsilon_{i j 3}$.

REMARK 1. Since the vertical projection of the angular velocity $\omega_{z}=(\boldsymbol{\omega} \cdot \gamma)$ remains unchanged and does not appear in the system (1.7), the behavior of the other quantities describing the motion of the ball does not depend on its value. This implies, in particular, that the motion of a rubber ball with the additional constraint $\omega_{z}=(\boldsymbol{\omega} \cdot \gamma)=0$ [8] is a particular case of the system of interest. The situation where the phase flow of one (nonholonomic) system is a particular case of a more general (Hamiltonian) system on a specific integral surface is also considered in [9].

\section{Explicit integration}

The eigenvalues $\lambda_{j}$ of the matrix $\mathbf{L}$ satisfy the equation

$$
\lambda^{2} P_{4}(\lambda)=0, \quad P_{4}(\lambda)=\left(\frac{\lambda}{\mu}\right)^{4}+4\left(\frac{\lambda}{\mu}\right)^{3}+4\left(\frac{\lambda}{\mu}\right)^{2}+\varepsilon^{2},
$$

where we have introduced the following notation to abbreviate the formulae:

$$
\mu=\frac{\mu_{0}(k+1)}{2 k}>0, \quad \varepsilon=\frac{\mu_{0}}{\mu^{2}}=\frac{4 k^{2}}{\mu_{0}(k+1)^{2}}>0 .
$$

Equation (2.1) has two zero roots which correspond to two linearly independent eigenvectors forming the kernel of the matrix $\mathbf{L}$ :

$$
\boldsymbol{e}_{1}=(1,0,0,0,1,0), \quad \boldsymbol{e}_{2}=(0,1,0,0,0,1) .
$$

In this case there exists a family of fixed points of the system (1.7)

$$
\boldsymbol{z}_{c}=x_{c} \boldsymbol{e}_{1}+y_{c} \boldsymbol{e}_{2}=\left(x_{c}, y_{c}, 0,0, x_{c}, y_{c}\right),
$$

where $x_{c}$ and $y_{c}$ are independent parameters defining this family.

For fixed $\left(x_{c}, y_{c}\right)$ a fixed point of this family corresponds to a state of the system in which the center of mass of the ball does not move relative to the fixed reference system. In this case, the plane rotates under it so that the ball rolls without slipping. That is to say, the ball has a constant angular velocity that (in dimensional form) is related to the angular velocity $\Omega$ of rotation of the plane by

$$
\omega_{1}=\frac{x_{c} \Omega}{R}, \quad \omega_{2}=\frac{y_{c} \Omega}{R} .
$$


The nonzero roots of the characteristic polynomial (2.1), i.e., the roots of the polynomial $P_{4}(\lambda)$, are pairwise complex conjugate and have the form

$$
\begin{aligned}
& \lambda_{1,2}=\mu(-1+\sqrt{1 \pm i \varepsilon})=\alpha_{1} \pm i \beta \\
& \lambda_{3,4}=\mu(-1-\sqrt{1 \pm i \varepsilon})=\alpha_{2} \pm i \beta
\end{aligned}
$$

where $i$ is a complex unit. The real parts $\alpha_{1}, \alpha_{2}$ and the imaginary part $\beta$ of the roots of the polynomial $P_{4}(\lambda)$ have the form

$$
\begin{gathered}
\alpha_{1}=\frac{\mu}{2}\left(\sqrt{2+2 \sqrt{1+\varepsilon^{2}}}-2\right), \quad \alpha_{2}=\frac{\mu}{2}\left(-\sqrt{2+2 \sqrt{1+\varepsilon^{2}}}-2\right), \\
\beta=\frac{\mu}{\sqrt{2}} \sqrt{\sqrt{1+\varepsilon^{2}}-1} .
\end{gathered}
$$

We see that, according to (2.2) and (2.5), the following inequalities are satisfied (see Fig. 2):

$$
\alpha_{2}<0<\alpha_{1} .
$$

Thus, the fixed point $\boldsymbol{z}_{c}(2.3)$ is an unstable focus.

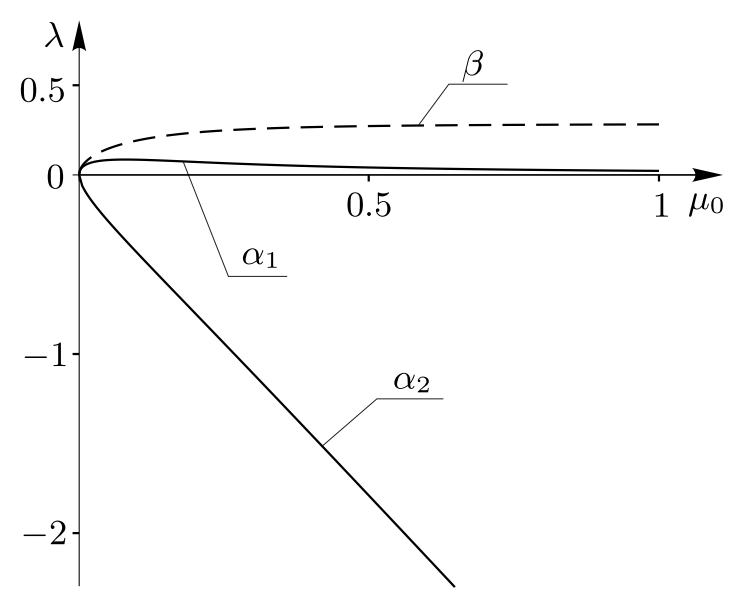

Fig. 2. Dependence of the real parts $\left(\alpha_{1}, \alpha_{2}\right)$ and the imaginary part $(\beta)$ of the eigenvalues (2.4) on the coefficient $\mu_{0}$ at $k=2 / 5$.

According to (2.4), the solution $\boldsymbol{z}(t)$ of the system (1.7) is the sum:

$$
\begin{gathered}
\boldsymbol{z}(t)=\boldsymbol{z}_{0}+\boldsymbol{z}_{+}+\boldsymbol{z}_{-}, \\
\boldsymbol{z}_{0}=x_{0} \boldsymbol{e}_{1}+y_{0} \boldsymbol{e}_{2}, \quad \boldsymbol{z}_{+}=e^{\alpha_{1} t}\left(\boldsymbol{C}_{1} e^{i \beta t}+\boldsymbol{C}_{2} e^{-i \beta t}\right), \quad \boldsymbol{z}_{-}=e^{\alpha_{2} t}\left(\boldsymbol{C}_{3} e^{i \beta t}+\boldsymbol{C}_{4} e^{-i \beta t}\right),
\end{gathered}
$$

where $x_{0}, y_{0}$ are the constants defined from the initial conditions, $\boldsymbol{C}_{j}, j=1 \ldots 4$ are the complex constant vectors defined in terms of the eigenvectors of the matrix $\mathbf{L}$ and from the initial conditions $\boldsymbol{z}(0)$. Within the framework of this paper we do not present them explicitly, since they are cumbersome in form. We present only the expressions for $x_{0}, y_{0}$, which are expressed in terms of the initial values of linear $v_{x}(0), v_{y}(0)$ and angular $\omega_{x}(0), \omega_{y}(0)$ velocities:

$$
x_{0}=\frac{k \omega_{x}(0)-v_{y}(0)}{k}, \quad y_{0}=\frac{k \omega_{y}(0)-v_{x}(0)}{k} .
$$

According to (2.6) and (2.7), the solution of the system (1.7) is the sum of the constant term $\left(\boldsymbol{z}_{0}\right)$ and the oscillating terms $\left(\boldsymbol{z}_{+}\right.$and $\left.\boldsymbol{z}_{-}\right)$. The frequency of oscillations of these terms 
is the same and equals $\beta$, the amplitudes are monotonic increasing $\left(e^{\alpha_{1} t}\right)$ and decreasing $\left(e^{\alpha_{2} t}\right)$ functions.

A characteristic time dependence of the increasing $x_{+}$and decreasing $x_{-}$terms in the solution for the function $x(t)$ (the first coordinate of the vector $\boldsymbol{z}$ ) at different coefficients $\mu_{0}$ is presented in Fig. 3. It can be seen that under these initial conditions the decreasing term $\left(x_{-}\right)$gives a contribution to the general solution only at the initial stage, for the time that does not exceed the period of one complete oscillation of the coordinate $x(t)$. We show that the solution (2.7) is analogous in the general case as well.

To this end we calculate how many times $N$ the amplitude of oscillations $e^{\alpha_{2} t}$ of the term $\boldsymbol{z}_{-}$ in one period $T=2 \pi / \beta$ will decrease:

$$
N=\exp \left(\frac{2 \pi\left|\alpha_{2}\right|}{\beta}\right)
$$

According to (2.5), the ratio in the exponent is

$$
\frac{\left|\alpha_{2}\right|}{\beta}=\frac{\sqrt{1+\sqrt{1+\varepsilon^{2}}}+1}{\sqrt{\sqrt{1+\varepsilon^{2}}-1}}>1 \text {. }
$$

Therefore, for one period of oscillation the amplitude of the term $\boldsymbol{z}_{-}$decreases $N>\exp (2 \pi) \approx$ 535 times. Thus, as shown in the example in Fig. 3, the term $\boldsymbol{z}_{-}$decreases to insignificant values in a time interval less than one period of oscillations.

Thus, under given initial conditions the solution of the system (1.7) is defined, except for a short initial period, by the increasing terms $\boldsymbol{z}_{+}$. This corresponds to the motion of the center of mass of the ball in a monotonically untwisting spiral around the projection of point $\boldsymbol{z}_{0}$ onto the plane $O x y$. Examples of trajectories of the center of mass of the ball for different coefficients of friction are given in Fig. 4.

The motion described above is similar to that of the ball on the plane if one incorporates the moment of resistance to rolling which is proportional to the angular velocity of the ball [2]. But in this case the untwisting of the spiral occurs much faster (see also [11]).
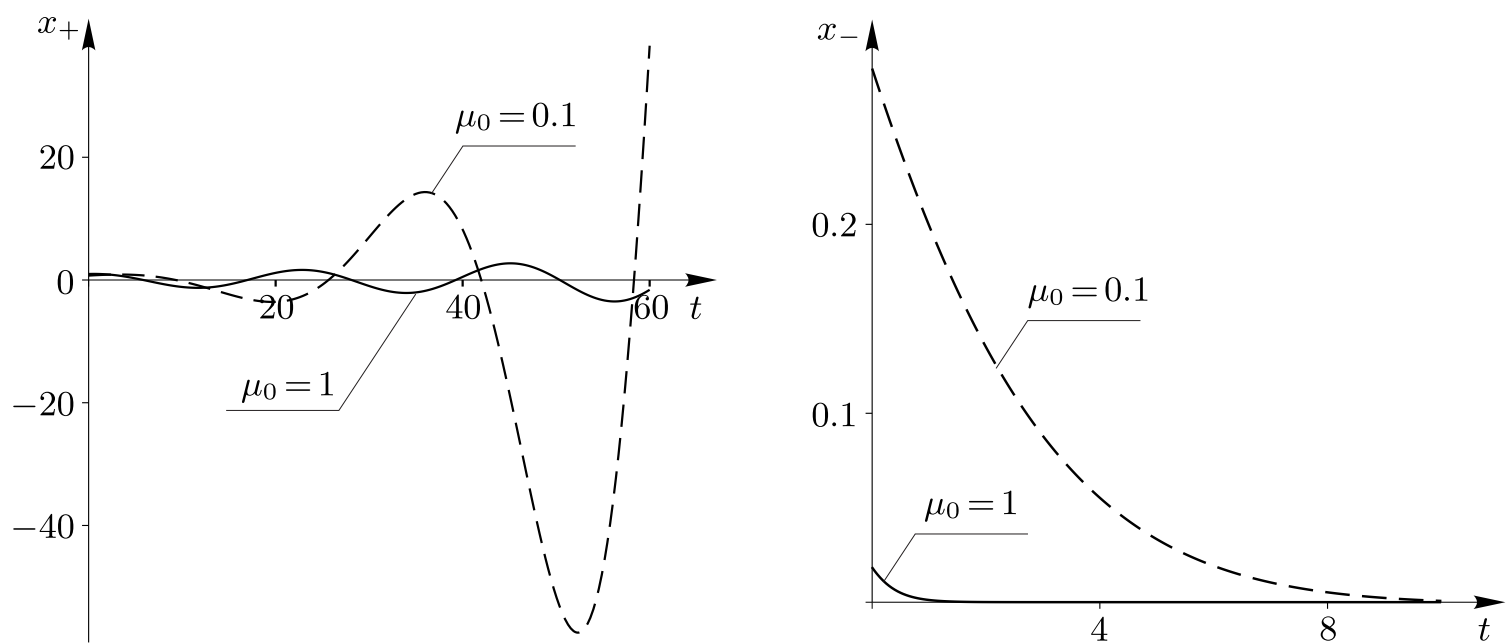

Fig. 3. A characteristic dependence of the increasing $\left(x_{+}\right)$and decreasing $\left(x_{-}\right)$terms of the solution for the function $x(t)$, which are constructed for $\mu_{0}=0.1$ and $\mu_{0}=1, k=2 / 5$ and the initial values of the coordinates and velocities $x(0)=1, y(0)=1, v_{x}(0)=0, v_{y}(0)=0, \omega_{x}(0)=0, \omega_{y}(0)=0$. 


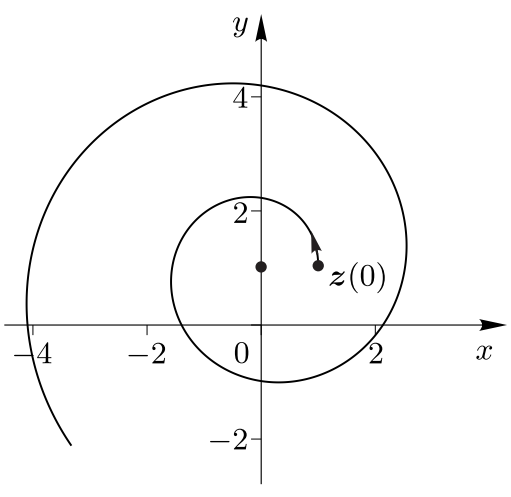

$\mu_{0}=0.5$

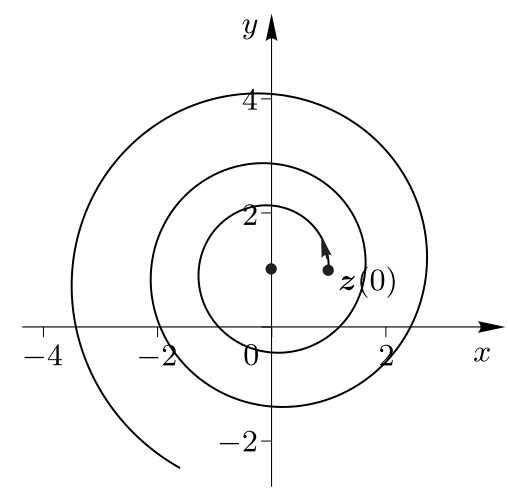

$\mu_{0}=1$

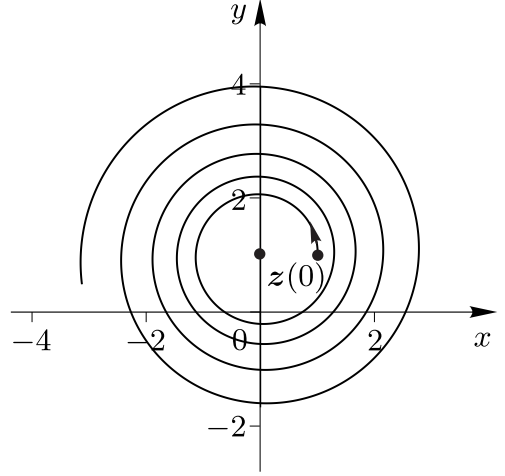

$\mu_{0}=2$

Fig. 4. Trajectories of the center of mass of a ball moving with slipping on a rotating plane, under the initial conditions $x(0)=1, y(0)=1, v_{x}(0)=0, v_{y}(0)=0, \omega_{x}(0)=0, \omega_{y}(0)=1$, for different coefficients of friction. The heavy dot denotes the position of the point $\left(x_{0}, y_{0}\right)(2.8)$, and $\boldsymbol{z}(0)$ is the projection of the vector $\boldsymbol{z}$ onto the plane $O x y$ at the initial time instant.

On the other hand, as the coefficient of friction $\mu_{0}$ increases, the velocity of untwisting of the spiral decreases appreciably (see Fig. 4). That is, one can assume that in the limiting case $\mu_{0} \rightarrow \infty$ the trajectory will correspond to the nonholonomic rolling model in which the ball moves in closed circles with a constant frequency determined only by the radius of inertia of the ball [2]. We consider this limiting case in more detail in the next section.

\section{The limiting case of the friction coefficient}

In this section we analyze the solution of the system (1.7) for $\mu_{0} \rightarrow \infty$.

We note that, according to $(2.2), \varepsilon \rightarrow 0$ as $\mu_{0} \rightarrow \infty$.

Let us expand the expression in brackets (2.4) in a power series of the small parameter $\varepsilon$ and represent the roots $\lambda$ of the characteristic equation in the form

$$
\begin{gathered}
\lambda_{1,2}=\mu\left( \pm \frac{i \varepsilon}{2}+\frac{\varepsilon^{2}}{8}+O\left(\varepsilon^{3}\right)\right), \\
\lambda_{3,4}=\mu\left(-2 \pm \frac{i \varepsilon}{2}-\frac{\varepsilon^{2}}{8}+O\left(\varepsilon^{3}\right)\right) .
\end{gathered}
$$

Substituting (2.2) into (3.1), we obtain

$$
\begin{gathered}
\lambda_{1,2}= \pm \frac{i k}{k+1}+\frac{k^{3}}{\mu_{0}(k+1)^{3}}+O\left(\frac{1}{\mu_{0}^{2}}\right) \\
\lambda_{3,4}=-\frac{\mu_{0}(k+1)}{k} \pm \frac{i k}{k+1}+\frac{k^{3}}{\mu_{0}(k+1)^{3}}+O\left(\frac{1}{\mu_{0}^{2}}\right) .
\end{gathered}
$$

Thus, for large $\mu_{0}$ the general solution (2.7) can now be represented as

$$
\begin{aligned}
\boldsymbol{z}(t)= & \boldsymbol{z}_{0}+\boldsymbol{D}_{1} \exp \left(-\frac{\mu_{0}(k+1) t}{k}\right)+ \\
& +\exp \left(\frac{k^{3} t}{\mu_{0}(k+1)^{3}}\right)\left(\boldsymbol{D}_{2} \cos \left(\frac{k}{k+1} t\right)+\boldsymbol{D}_{3} \sin \left(\frac{k}{k+1} t\right)\right),
\end{aligned}
$$


where $\boldsymbol{D}_{1}, \boldsymbol{D}_{2}, \boldsymbol{D}_{3}$ are the real constant vectors defined in terms of the eigenvectors of the matrix $\mathbf{L}$ and from the initial conditions $\boldsymbol{z}(0)$.

Thus, on each fixed time interval, as $\mu_{0} \rightarrow \infty$ the solution (3.3) tends to the function

$$
\boldsymbol{z}(t)=\boldsymbol{z}_{0}+\boldsymbol{D}_{2} \cos \left(\frac{k}{k+1} t\right)+\boldsymbol{D}_{3} \sin \left(\frac{k}{k+1} t\right),
$$

which describes exactly the rolling of a homogeneous ball in the case of a nonholonomic dissipation-free model of motion $[1,2,10]$.

Thus, the nonholonomic constraint that arises in the case of a ball rolling on a rotating plane can be realized by means of viscous friction forces and corresponds to the case where the coefficient of viscous friction is equal to infinity.

The author extends her gratitude to A. V. Borisov, I. S. Mamaev and A. A. Kilin for the formulation of the problem and fruitful discussions of the results obtained.

\section{References}

[1] Earnshaw, S., Dynamics, or An Elementary Treatise on Motion, 3rd ed., Cambridge: Deighton, 1844.

[2] Borisov, A. V., Ivanova, T. B., Karavaev, Yu. L., and Mamaev, I. S., Theoretical and Experimental Investigations of the Rolling of a Ball on a Rotating Plane (Turntable), Eur. J. Phys., 2018, vol. 39, no. 6, 065001, $13 \mathrm{pp}$.

[3] Fufaev, N. A., A Sphere Rolling on a Horizontal Rotating Plane, J. Appl. Math. Mech., 1983, vol.47, no. 1, pp. 27-29; see also: Prikl. Mat. Mekh., 1983, vol.47, no. 1, pp.43-47.

[4] Fufaev, N.A., On the Possibility of Realizing a Nonholonomic Constraint by Means of Viscous Friction Forces, J. Appl. Math. Mech., 1964, vol. 28, no.3, pp.630-632; see also: Prikl. Mat. Mekh., 1964, vol. 28, no. 3, pp. 513-515.

[5] Kolesnikov, S.L., The Problem of a Homogeneous Heavy Ball Rolling with Slipping in a Vertical Cylinder, in Collection of scientific and Methodical Papers on Theoretical Mechanics: Vol. 17, Moscow: Vysshaya Shkola, 1986, pp. 118-121 (Russian).

[6] Karapetyan, A. V. and Rumyantsev, V. V., Stability of Conservative and Dissipative Systems, Itogi Nauki Tekh. Ser. Obshch. Mekh., vol.6, Moscow: VINITI, 1983 (Russian).

[7] Eldering, J., Realizing Nonholonomic Dynamics as Limit of Friction Forces, Regul. Chaotic Dyn., 2016, vol. 21, no. 4, pp. 390-409.

[8] Borisov, A. V. and Mamaev, I. S., Conservation Laws, Hierarchy of Dynamics and Explicit Integration of Nonholonomic Systems, Regul. Chaotic Dyn., 2008, vol. 13, no. 5, pp. 443-490.

[9] Borisov, A. V., Kilin, A. A., and Mamaev, I.S., The Hamilton Principle and the Rolling Motion of a Symmetric Ball, Dokl. Phys., 2017, vol.62, no.6, pp. 314-317; see also: Dokl. Akad. Nauk, 2017, vol. 474, no. 5, pp. 558-562.

[10] Gersten, J., Soodak, H., and Tiersten, M.S., Ball Moving on Stationary or Rotating Horizontal Surface, Am. J. Phys., 1992, vol.60, no. 1, pp. 43-47.

[11] Kyeong Min Kim, Donggeon Oh, Junghwan Lee, Young-Gui Yoon, Chan-Oung Park. Dynamics of Cylindrical and Spherical Objects on a Turntable HAL Id: hal - 01761333, version 1, https://hal.archives-ouvertes.fr/hal-01761333 (2018). 